\title{
Prognostic Significance of STC2 and DNA methylation in Head and Neck Squamous Cell Carcinoma: A Study Based on the TCGA and GEO Databases
}

\section{Guangyao Li}

Zhongshan Hospital

\section{Daquan Wu}

Zhongshan Hospital

\section{Lei Zhou}

Zhongshan Hospital

Dan You

Zhongshan Hospital

Xinsheng Huang ( $\nabla$ huang.xinsheng@zs-hospital.sh.cn )

Zhongshan Hospital

\section{Research Article}

Keywords: Stanniocalcin 2, Head and neck squamous cell carcinoma, Survival, Methylation, Immune infiltration

Posted Date: February 12th, 2021

DOl: https://doi.org/10.21203/rs.3.rs-191202/v1

License: (c) (i) This work is licensed under a Creative Commons Attribution 4.0 International License. Read Full License 


\section{Abstract}

Background: Head and neck squamous cell carcinoma (HNSC) is a popular malignancy type that brings about poor prognosis with a low survival rate worldwide. Stanniocalcin 2 (STC2) is a glycosylated peptide hormone and shows the potential to become a new biomarker for the evaluation of malignant tumors. The purpose of this study was to explore the prognostic implications of STC2 and DNA methylation in HNSC and the role of STC2 expression in immune cell infiltration.

Methods: STC2 gene expression data were collected from Gene Expression Omnibus (GEO) and the Cancer Genome Atlas (TCGA) databases. Univariate and multivariate analyses were employed to screen prognostic risk factors. The relationship between STC2 expression and TP53 mutation in HNSC was explored. TCGA data were utilized to analyze how STC2 expression affected immune cell infiltration in HNSC.

Results: STC2 was highly expressed in HNSC patients $(P<0.01)$, especially those with a lower overall survival rate $(P<0.0001)$. TP53 mutation might be a risk factor of STC2 overexpression in HNSC $(P=$ 0.0015). STC2 expression was negatively correlated with STC2 methylation (Spearman: $-0.43, P<0.001$ ). Hypermethylation or hypomethylation at the eight CpG sites most related to STC2 expression was identified as independent factors for HNSC prognosis. STC2 was positively correlated with cancerassociated fibroblasts infiltration and associated with the infiltration of various immune cells.

Conclusion: STC2 can be regarded as a vital prognostic biomarker of HNSC due to its essential roles in immune cell infiltration.

\section{Background}

Head and neck squamous cell carcinoma (HNSC) arises in the mucous membranes of the oral cavity, oropharynx, and larynx. It is the sixth most malignancy across the world and contributes most to recurrent upper gastrointestinal tumors[1,2]. China has an incidence of approximately 135 cases per 100,000 people every year [3]. Defined as a solid tumor, HNSC exhibits high invasiveness and links to poor clinical outcomes due to local recurrence, regional or distant metastasis [4]. Fortunately, few decades of progress in chemotherapy, radiotherapy, and surgical treatment for HNSC have somewhat improved the five-year survival rate [5]. These encourage further exploration of sensitive and effective biomarkers, also serving as therapeutic targets, of the disease. Among published candidates, STC2 is more prominent in predicting the occurrence and development of assorted tumor types $[6,7]$.

STC2, first discovered in a bony fish, is a glycosylated peptide hormone and essential in calcium and phosphorus homeostasis. In mammals, it is ubiquitously present in multiple tissues and involved in glutamine or glucose deprivation and is overexpressed under hypoxia, endoplasmic reticulum stress, and radiation[8-11]. The microenvironment characterized by hypoxia, low glucose and glutamine supply promotes the occurrence of several solid tumors. Tumor cells exploit STC2 overexpression to become more resilient to such a hostile condition and more proliferative for progression. The latest evidence 
reveals aberrant STC2 expression in assorted tumor types, which can be considered a new biomarker for prognosis prediction. Recent studies also linked STC2 to a poor prognosis of patients with gastric, colorectal, pancreatic, breast cancers, and other malignancies [6, 12-15]. All these suggest that aberrant STC2 expression is closely related to the occurrence, progression, clinical manifestations, and prognosis of tumors. Kita Y, Law AY et al. further confirmed that STC2 could be a predictor for lymph node metastasis of esophageal squamous cell carcinoma and that it promoted epithelial-mesenchymal transition (EMT) of ovarian cancer cells under hypoxia $[11,16]$. But little is known about the value of STC2 in predicting HNSC prognosis and related manifestations. Therefore, we mined the Gene Expression Omnibus (GEO) and the Cancer Genome Atlas (TCGA) databases to explore whether high STC2 expression and DNA methylation were associated with HNSC progression and whether STC2 expression was involved in immune cell infiltration in HNSC.

\section{Materials}

\section{Microarray data processing}

The microarray datasets covering HNSC, or combined with laryngeal squamous cell carcinoma(LSCC), were downloaded from the NCBI GEO database (http://www.ncbi.nlm.nih.gov/geo/). Then, gene expression data were selected from annotated probe sets. The probe set annotation mainly refers to the new version of the annotation file downloaded from the Affymetrix website (http://www.affymetrix.com/support/technical/byproduct.affx). We also extracted TCGA survival data of HNSC patients from cBioPortal for Cancer Genomics (https://www.cbioportal.org/). The Limma package (http://bioconductor.org/packages/release/bioc/html/limma.html) was employed to identify differentially expressed genes (DEGs) from four datasets, GSE143224 and GSE59102 (adj. P. val $\leq 0.001$ and $|\log F C| \geq 1$ ), GSE51985 (adj. P. val $\leq 0.05$ and $|\log F C| \geq 1$ ), and the TCGA cohort (BH adjusted Pvalue $\leq 0.05$ ), respectively, to screen out overlapping genes. Ultimately, STC2 was confirmed and further explored based on previous studies.

\section{Data mining of public databases}

First, we used the online tools gene expression profiling interactive analysis (GEPIA)(http://gepia.cancerpku.cn/index.html)[17] and UALCAN (http://ualcan.path.uab.edu/analysis.html)[18] to validate aberrant STC2 expression in HNSC. The association of high STC2 expression with the overall survival (OS) of HNSC patients was assessed using the TCGA cohort. Subsequently, the correlation analysis of STC2 expression and TP53 mutation was performed using the cBioPortal platform. We mined the Human Protein Atlas (HPA )database(https://www.proteinatlas.org)[19] to understand whether differentially expressed SCT2 was linked to immunohistochemistry (IHC) results in HNSC patients. The intensity of IHC staining was scored as 0 (no staining), 1 (weak staining), and 2 (strong staining), corresponding to the frequency of immune-reactive tumor cells $(0-25 \%, 26-75 \%, 76-100 \%)$. UCSC Xena (http://xena.ucsc.edu/) enabled analysis for the prognostic value of STC2 methylation in HNSC.

\section{Immune infiltration analysis}


Timer2.0 offers comprehensive assessments for the association between immune infiltration and STC2 gene features in HNSC [20]. We analyzed the crosstalk between STC2 expression and cancer-associated fibroblasts (CAFs) using the TIMER, TIDE, XCELL, MCPCOUNTER, and EPIC algorithms to assess immune infiltration levels. The Partial correlation coefficient (partial corr.) and the corresponding P-value were yielded via the Spearman rank correlation test following purity adjustment. The data were visualized as heat maps and scatter plots. In addition, the correlation module was utilized to evaluate the association between STC2 and tumor-infiltrating immune cells, including T cells (general), CD8 T cells, B cells, M1 cells, and M2 cells, in HNSC, as described in our previous studies [21, 22].

\section{Enrichment analysis of STC2-related genes}

We searched the STRING website (https://string-db.org/) [23] for proteins interacting with STC2 protein, with an interaction cutoff of 0.4 . The STC2 protein-protein interaction (PPI) data were clustered using a clustering method. The top 102 genes positively associated with STC2 in HNSC were analyzed using UALCAN, and genes with extremely low expression (median TPM $<0.5$ ) were ruled out. Then KEGG (Kyoto encyclopedia of genes and genes) pathway analysis and GO (gene ontology) enrichment analysis were performed on these genes in R [R-3.6.1,64 bit](https://www.r-project.org/).

\section{Results}

\section{Clinical and prognostic implication of STC2 overexpression in HNSC}

We analyzed STC2 expression in HNSC using GSE143112, GSE51985, and GSE59102 cohorts. The results showed high STC2 expression in HNSC (or combined with LSCC) (Fig. 1), which was subsequently confirmed with the TCGA cohort using the GEPIA and UALCAN web-tools (Fig. 2a,b). STC2 expressions in stage 1, 2, 3, 4 HNSC significantly increased versus normal controls (all $P<0.001$ ) (Fig. 2c). The Kaplan Meier curves and Cox proportional hazards regression model analysis for its correlation with survival prediction revealed that high STC2 expression was a prognostic risk factor of worse OS in HNSC (Table 1, Fig. 2d). The cBioPortal cancer genomic analysis offered clear alterations that the frequency of TP53 mutation was about 70\% in HNSC and that STC2 expression was markedly upregulated in the mutated TP53 group $(P=0.0015)$ (Fig. 3a,b). This suggested a close relationship between aberrant STC2 expression and TP53 mutation in HNSC. So we further mined the HPA database for STC2 protein expression profiles. By comparing the intensity of IHC staining between STC2-positive HNSC tissues versus normal controls, we found that STC2-positive staining was stronger in HNSC tissues than normal ones(Fig. 3c,d), further demonstrating STC2 overexpression in HNSC. 
Table 1

Univariate and multivariate analyses of associations between clinicopathological components and STC2 expression

\begin{tabular}{|c|c|c|c|c|c|c|}
\hline \multirow[t]{2}{*}{ Characteristics } & \multicolumn{3}{|c|}{ Univariate Cox } & \multicolumn{3}{|c|}{ Multivariate Cox } \\
\hline & $\begin{array}{l}\text { Hazard } \\
\text { ratio }\end{array}$ & $95 \% \mathrm{Cl}$ & $\begin{array}{l}\mathrm{P}- \\
\text { value }\end{array}$ & $\begin{array}{l}\text { Hazard } \\
\text { ratio }\end{array}$ & $95 \% \mathrm{Cl}$ & $\begin{array}{l}\mathrm{P}- \\
\text { value }\end{array}$ \\
\hline Age ( $\geq$ median vs. $<$ median) & 1.39 & $\begin{array}{l}1.05- \\
1.84\end{array}$ & 0.0212 & 1.23 & $\begin{array}{l}0.893- \\
1.70\end{array}$ & 0.2036 \\
\hline Sex (male vs. female) & 1.29 & $\begin{array}{l}0.965- \\
1.73\end{array}$ & 0.0853 & & & \\
\hline Stage (III and IV vs. I and II) & 2 & $\begin{array}{l}1.32- \\
3.01\end{array}$ & $\dot{0} 001$ & 1.86 & $\begin{array}{l}0.919- \\
3.75\end{array}$ & 0.0848 \\
\hline $\mathrm{T}$ (T3 and T4 vs. T1 and T2) & 1.98 & $\begin{array}{l}1.44- \\
2.73\end{array}$ & $\hat{0} .001$ & 1.59 & $\begin{array}{l}0.990- \\
2.56\end{array}$ & 0.0552 \\
\hline $\begin{array}{l}\mathrm{N}(\mathrm{N} 3 \text { and } \mathrm{N} 4 \text { vs. } \mathrm{N} 1 \text { and } \\
\mathrm{N} 2)\end{array}$ & 2.85 & $\begin{array}{l}1.26- \\
6.46\end{array}$ & 0.012 & 2.28 & $\begin{array}{l}0.997- \\
5.20\end{array}$ & 0.051 \\
\hline M (M1 vs. M0) & 21.9 & $\begin{array}{l}2.74- \\
175\end{array}$ & 0.0036 & 16 & $2.14-98$ & 0.0063 \\
\hline HPV_STATUS_ISH (+ vs. -) & 1.71 & $0.834-3.5$ & 0.1429 & & & \\
\hline HPV_STATUS_P16 (-vs. +) & 1.32 & $\begin{array}{l}0.718- \\
2.43\end{array}$ & 0.371 & & & \\
\hline PATH_MARGIN (+ vs. -) & 1.14 & $\begin{array}{l}0.812- \\
1.61\end{array}$ & 0.4431 & & & \\
\hline $\begin{array}{l}\text { PERINEURAL_INVASION (+ } \\
\text { vs. -) }\end{array}$ & 1.33 & $\begin{array}{l}0.948- \\
1.86\end{array}$ & 0.0986 & & & \\
\hline $\begin{array}{l}\text { Grade ( } 3 \text { and } 4 \text { vs. } 1 \text { and } 2) \\
(+ \text { vs }-)\end{array}$ & 1.03 & $\begin{array}{l}0.75- \\
1.41\end{array}$ & 0.858 & & & \\
\hline $\begin{array}{l}\text { STC2 }(\geq \text { median vs }< \\
\text { median) }\end{array}$ & 1.84 & $\begin{array}{l}1.39- \\
2.42\end{array}$ & $<.001$ & 1.6 & $\begin{array}{l}1.149- \\
2.22\end{array}$ & 0.0043 \\
\hline
\end{tabular}

\section{Analysis of STC2 DNA methylation}

As expected, the Heatmap demonstrated that STC2 expression was negatively correlated with STC2 DNA methylation ( $r=-0.43, P<0.001)$ (Fig. 4a,b), and the latter were identified at $33 \mathrm{CpG}$ sites (Fig. 4c). Next, we estimated methylation levels at the CpG sites affecting STC2 mRNA expression using Spearman's rank correlation coefficient. As illustrated in Fig. 4d, four CpG sites (cg19673350, cg18335607, cg02135395, and cg06439005) were most negatively correlated with STC2 expression, and another four (cg01984743, cg26605683, cg15420720, and cg03846076) most positively correlated with STC2 expression were confirmed. Kaplan-Meier survival curves were plotted based on the methylation levels at the two groups 
of CpG sites (Fig. 5). The Kaplan-Meier analysis for the negative-correlation group revealed that HNSC patients with $\mathrm{CpG}$ demethylation was associated with worse OS. The survival curves for the positivecorrelation group revealed that $\mathrm{CpG}$ hypermethylation predicted worse OS of HNSC patients. These results further implied that high STC2 expression predicted a poor prognosis of HNSC patients.

\section{Relationship between STC2 and immune cells}

Tumor-infiltrating immune cells are important components in the tumor microenvironment, closely associated with tumor initiation, development, and metastasis [24-27]. We searched the TIMER2.0 database and assessed the association between STC2 mRNA expression and immune cell infiltration. CAFs as a component of the tumor-stromal compartment predominantly regulate the function of multiple tumor-infiltrating immune cells[28]. We estimated the relationship between the CAF infiltration level and STC2 expression in HNSC using the TIMER, TIDE, XCELL, MCPCOUNTER, and EPIC algorithms. The results showed that the CAF infiltration level was significantly and positively correlated with STC2 expression (Fig. 6a). Each STC2 expression matrix where STC2 expression was linked to CAFs/tumorinfiltrating immune cells was analyzed with one algorithm after adjusting for tumor purity (Fig. 6b). By using the TIDE algorithm, the analysis showed that STC2 expression was positively associated with the CAF infiltration level $(r=0.413, P=1.18 \mathrm{e}-21)$. This correlation was confirmed by analysis using other algorithms. Furthermore, we analyzed associations of STC2 expression with tumor-infiltrating immune cells in HNSC, including T (general) cells, CD8 T cells, B cells, M1 cells, M2 cells. STC2 expression was negatively associated with the infiltration levels of T (general) cells, CD8 T cells, and B cells and positively associated with those of M1 and M2 cells (Fig. 7). From the above results, it can be concluded that STC2 expression is closely linked to immune cell infiltration in HNSC, and high STC2 expression can predict worse OS of patients. We therefore speculated that immune infiltration could be one of the mechanisms contributing to STC2's impacts on HNSC prognosis.

\section{Enrichment analysis of STC2-related genes}

We initially screened 102 genes closely related to aberrant STC2 expression in HNSC using TCGA data with the UALCAN web-tool. We then implemented KEGG and GO enrichment analyses to explore the underlying mechanisms of STC2's prediction of HNSC prognosis. The results showed that these hub genes were most enriched in "metabolic pathways" and "biosynthesis of antibodies" based on KEGG analysis (Fig. 8a). Meanwhile, "oxidation-reduction process" and "positive regulation of cell proliferation" were top GO terms significantly associated with these genes (Fig. 8a). The top 10 proteins interacting with STC2 were illustrated in Fig. 8b.

\section{Discussion}

HNSC is the sixth most common non-skin cancer worldwide, with an overall incidence of 600,000 cases per year and a mortality rate of 50\%[29]. STC2 gene encodes a secreted glycoprotein abundant in various tissues with an autocrine/paracrine function. Yoshiaki Kita et al. suggested that high STC2 expression in tumor tissues sensitively predicted shorter five-year survival [16]. The upregulation of STC2 expression 
has been proven in other tumor types, for example, colorectal and prostate cancers [30,31]. It may be associated with clinical features of HNSC progression, such as tumor proliferation and lymph node invasion, and a worse prognosis [16]. All these are consistent with our findings. Our results also demonstrated that the STC2 gene was differentially expressed between normal and stage 1-4 HNSC patients, respectively, and this difference was more evident in patients with higher clinical stage. Therefore, we speculated that STC2 was involved in HNSC progression.

TP53 protein regulates the expressions of a large number of target genes and encodes $p 53$. $P 53$ has been considered an oncogene early on as it is preferentially expressed in cancerous cells [32]. Since 1989 when Levine et al. found wild-type $p 53$ as a tumor suppressor gene [33], it has captured more attention and has been confirmed as a regulator of several cellular activities, such as apoptosis, proliferation, differentiation, and cell-cycle control [34]. Its multi-function role has been observed in DNA damage, hypoxia, oxidative stress, DNA mutations, nutrient deprivation, and other stress responses. Alterations in TP53 gene expression, needless to say, are most certain to be detected in a significant proportion of human cancers. Most studies identified TP53 mutations in exons 5-8 with missense changes, but some ascertained functional mutations in exons $5-8$ of TP53, which actively contributed to cancer progression $[35,36]$. Evidence about TP53 mutations generally supports their associations with shorter recurrencefree survival or OS of HNSC patients [37-39]. This relationship has subsequently been ascertained by a clinical trial comparing TP53-mutated versus wild-type TP53 patients with HNSC [39]. Our study found that the frequency of TP53 mutations in HNSC was approximately $70 \%$, and STC2 expression was pronouncedly increased in the mutated TP53 group. Therefore, the association between TP53 mutations and high STC2 expression is more likely to affect HNSC occurrence and development.

Mammalian DNA methylation is essential in the epigenetic regulation of gene transcription. Dysregulation of genomic DNA methylation has been found to participate in carcinogenesis [40]. A previous study reported that STC2 silencing in human cancer cells could be associated with the methylation of $\mathrm{CpG}$ islands of STC2. Methylation of the STC2 promoter is a potential mechanism to hinder STC2 expression in cancer cells[41]. Hence, we explored the relationship between STC2 expression and methylation in HNSC, and consistently, the results suggested a negative correlation between them. Moreover, the eight CpG sites revealing a positive and negative correlation with STC2 expression were classified into two groups, respectively. Kaplan-Meier survival curves for the negative-correlation group indicated the association between a lower methylation level and high STC2 expression. The survival curves for the positive-correlation group showed CpG hypermethylation predicted higher STC2 expression, suggesting worse OS of patients.

Numerous studies of STC2 expression within the tumor microenvironment found that STC2 enhanced tumor cell migration and invasion [42, 43]. Clinical studies confirmed STC2 as a new biomarker of multiple cancers due to its involvement in tumor neovascularization $[44,45]$. STC is known for its potent anti-inflammatory effect [46, 47], of which STC1 exerts the effect via inducing uncoupling proteins to reduce oxidative stress [48]. STC2, homologous to STC1, is activated by oxidative stress to forestall cell apoptosis. The present study revealed a positive correlation between STC2 expression and the infiltration 
levels of CAFs, indicating that STC2 may promote fibrogenesis in HNSC. Previous studies have reported that STC2, as an immune-related protein, is a potential oncoprotein in the process of

hepatocarcinogenesis and is considered a promising biomarker and molecular target[49,50]. It is reported that STC2 can inhibit CD 8+T cell infiltration [51]. These are consistent with our conclusions.

Our study offered KEGG and GO enrichment analyses based on initially screened genes related to aberrant STC2 expression in HNSC and identified "metabolic pathways" and "biosynthesis of antibodies" as highly relevant pathway terms of STC2's role in head and neck carcinogenesis. Also, "oxidationreduction process" and "positive regulation of cell proliferation" were highly associated with the functions of the STC2 gene in this process.

Besides, three limitations are deserving to be discussed. First, inevitably, the raw data from the GEO and TCGA databases were originally used for other purposes. So we have been unable to validate the prognostic value of STC2 gene knockdown or protein downexpression in HNSC. Second, although we preliminarily analyzed the biological processes of STC2 in HNSC using enrichment analysis, the specific mechanism of how STC2 expression/methylation patterns affect HNSC progression requires more research support. Finally, the details of how STC2 promotes immune cell infiltration in HNSC are worthy of standardized investigation. However, the current results are encouraging, considering STC2 as a promising biomarker for the predicting HNSC prognosis.

\section{Conclusions}

STC2 is overexpressed in HNSC and negatively regulated by DNA methylation in HNSC. High STC2 expression, $\mathrm{CpG}$ demethylation negatively correlated with STC2 expression, CpG hypermethylation positively correlated with STC2 expression may predict a poor prognosis of HNSC patients. STC2 overexpression may contribute to the infiltration of T cells, B cells, and macrophages in HNSC. Overall, STC2 overexpression promotes immune cell infiltration probably via negative regulation of DNA methylation, showing the promising biomarker potential for HNSC prognosis.

\section{Abbreviations}

HNSC: Head and neck squamous cell carcinoma

STC2: Stanniocalcin 2

GEO: Gene Expression Omnibus

TCGA: The Cancer Genome Atlas

EMT: Epithelial-Mesenchymal Transition

LSCC: Laryngeal squamous cell carcinoma 
DEGs: Differentially expressed genes

OS: Overall survival

IHC: Immunohistochemistry

CAFs: Cancer-associated fibroblasts

PPI: Protein-protein interaction

KEGG: Kyoto encyclopedia of genes and genes pathway analysis

GO: Gene ontology

HPA: Human Protein Atlas

\section{Declarations}

\section{- Ethics approval and consent to participate:}

Not applicable.

\section{- Consent for publication:}

Not applicable.

\section{- Availability of data and materials:}

The datasets analysed during the current study are available in the [GEO and TCGA] repository,

1.[https://www.ncbi.nlm.nih.gov/geo/query/acc.cgi?acc=GSE143224];

2.[https://www.ncbi.nlm.nih.gov/geo/query/acc.cgi?acc=GSE51985];

3.[https://www.ncbi.nlm.nih.gov/geo/query/acc.cgi?acc=GSE59102];

4.[https://www.cbioportal.org/];

\section{- Competing interests:}

The authors declare that they have no competing interests.

\section{- Funding:}

Not applicable. 


\section{- Authors' contributions:}

Guangyao Li wrote the main manuscript text. Daquan Wu and Lei Zhou prepared figures 1-4 and table 1. Dan You prepared figures 5-8. Xinsheng Huang designed this work and substantively revised it. All authors reviewed the manuscript.

\section{- Acknowledgements:}

Not applicable.

\section{References}

1. Childs G, Fazzari M, Kung G, Kawachi N, Brandwein-Gensler M, McLemore M, Chen Q, Burk RD, Smith RV, Prystowsky MB et al: Low-level expression of microRNAs let-7d and miR-205 are prognostic markers of head and neck squamous cell carcinoma. Am J Pathol 2009, 174(3):736-745.

2. Yang S, Ji Q, Chang B, Wang Y, Zhu Y, Li D, Huang C, Wang Y, Sun G, Zhang L et al: STC2 promotes head and neck squamous cell carcinoma metastasis through modulating the PI3K/AKT/Snail signaling. Oncotarget 2017, 8(4):5976-5991.

3. Chen W, Zheng R, Baade PD, Zhang S, Zeng H, Bray F, Jemal A, Yu XQ, He J: Cancer statistics in China, 2015. CA Cancer J Clin 2016, 66(2):115-132.

4. Cao W, Liu JN, Liu Z, Wang X, Han ZG, Ji T, Chen WT, Zou X: A three-IncRNA signature derived from the Atlas of ncRNA in cancer (TANRIC) database predicts the survival of patients with head and neck squamous cell carcinoma. Oral Oncol 2017, 65:94-101.

5. Campbell JD, Yau C, Bowlby R, Liu Y, Brennan K, Fan H, Taylor AM, Wang C, Walter V, Akbani R et al: Genomic, Pathway Network, and Immunologic Features Distinguishing Squamous Carcinomas. Cell Rep 2018, 23(1):194-212 e196.

6. Lin C, Sun L, Huang S, Weng X, Wu Z: STC2 Is a Potential Prognostic Biomarker for Pancreatic Cancer and Promotes Migration and Invasion by Inducing Epithelial-Mesenchymal Transition. Biomed Res Int 2019, 2019:8042489.

7. He H, Qie S, Guo Q, Chen S, Zou C, Lu T, Su Y, Zong J, Xu H, He D et al: Stanniocalcin 2 (STC2) expression promotes post-radiation survival, migration and invasion of nasopharyngeal carcinoma cells. Cancer Manag Res 2019, 11:6411-6424.

8. Qie S, Liang D, Yin C, Gu W, Meng M, Wang C, Sang N: Glutamine depletion and glucose depletion trigger growth inhibition via distinctive gene expression reprogramming. Cel/ Cycle 2012, 11(19):3679-3690.

9. Ito D, Walker JR, Thompson CS, Moroz I, Lin W, Veselits ML, Hakim AM, Fienberg AA, Thinakaran G: Characterization of stanniocalcin 2, a novel target of the mammalian unfolded protein response with cytoprotective properties. Mol Cell Biol 2004, 24(21):9456-9469. 
10. Law AY, Wong CK: Stanniocalcin-2 is a HIF-1 target gene that promotes cell proliferation in hypoxia. Exp Cell Res 2010, 316(3):466-476.

11. Law AY, Wong CK: Stanniocalcin-2 promotes epithelial-mesenchymal transition and invasiveness in hypoxic human ovarian cancer cells. Exp Cell Res 2010, 316(20):3425-3434.

12. Ke J, Zhang BH, Li YY, Zhong M, Ma W, Xue H, Wen YD, Cai YD: MiR-1-3p suppresses cell proliferation and invasion and targets STC2 in gastric cancer. Eur Rev Med Pharmacol Sci 2019, 23(20):88708877.

13. Li Q, Zhou X, Fang Z, Pan Z: Effect of STC2 gene silencing on colorectal cancer cells. Mol Med Rep 2019, 20(2):977-984.

14. Wu J, Lai M, Shao C, Wang J, Wei JJ: STC2 overexpression mediated by HMGA2 is a biomarker for aggressiveness of high-grade serous ovarian cancer. Oncol Rep 2015, 34(3):1494-1502.

15. Jansen MP, Sas L, Sieuwerts AM, Van Cauwenberghe C, Ramirez-Ardila D, Look M, Ruigrok-Ritstier K, Finetti P, Bertucci F, Timmermans MM et al: Decreased expression of ABAT and STC2 hallmarks ERpositive inflammatory breast cancer and endocrine therapy resistance in advanced disease. $\mathrm{Mol}$ Oncol 2015, 9(6):1218-1233.

16. Kita Y, Mimori K, Iwatsuki M, Yokobori T, leta K, Tanaka F, Ishii H, Okumura H, Natsugoe S, Mori M: STC2: a predictive marker for lymph node metastasis in esophageal squamous-cell carcinoma. Ann Surg Oncol 2011, 18(1):261-272.

17. Tang Z, Li C, Kang B, Gao G, Li C, Zhang Z: GEPIA: a web server for cancer and normal gene expression profiling and interactive analyses. Nucleic Acids Res 2017, 45(W1):W98-W102.

18. Chandrashekar DS, Bashel B, Balasubramanya SAH, Creighton CJ, Ponce-Rodriguez I, Chakravarthi B, Varambally S: UALCAN: A Portal for Facilitating Tumor Subgroup Gene Expression and Survival Analyses. Neoplasia 2017, 19(8):649-658.

19. Uhlen M, Fagerberg L, Hallstrom BM, Lindskog C, Oksvold P, Mardinoglu A, Sivertsson A, Kampf C, Sjostedt E, Asplund A et al: Proteomics. Tissue-based map of the human proteome. Science 2015, 347(6220):1260419.

20. Li T, Fu J, Zeng Z, Cohen D, Li J, Chen Q, Li B, Liu XS: TIMER2.0 for analysis of tumor-infiltrating immune cells. Nucleic Acids Res 2020, 48(W1):W509-W514.

21. Danaher P, Warren S, Dennis L, D'Amico L, White A, Disis ML, Geller MA, Odunsi K, Beechem J, Fling SP: Gene expression markers of Tumor Infiltrating Leukocytes. J Immunother Cancer 2017, 5:18.

22. Yang J, Li H, Hu S, Zhou Y: ACE2 correlated with immune infiltration serves as a prognostic biomarker in endometrial carcinoma and renal papillary cell carcinoma: implication for COVID-19. Aging (Albany NY) 2020, 12(8):6518-6535.

23. Szklarczyk D, Morris JH, Cook H, Kuhn M, Wyder S, Simonovic M, Santos A, Doncheva NT, Roth A, Bork $P$ et al: The STRING database in 2017: quality-controlled protein-protein association networks, made broadly accessible. Nucleic Acids Res 2017, 45(D1):D362-D368.

24. Steven A, Seliger B: The Role of Immune Escape and Immune Cell Infiltration in Breast Cancer. Breast Care (Basel) 2018, 13(1):16-21. 
25. Fridman WH, Galon J, Dieu-Nosjean MC, Cremer I, Fisson S, Damotte D, Pages F, Tartour E, SautesFridman C: Immune infiltration in human cancer: prognostic significance and disease control. Curr Top Microbiol Immunol 2011, 344:1-24.

26. Fridman WH, Zitvogel L, Sautes-Fridman C, Kroemer G: The immune contexture in cancer prognosis and treatment. Nat Rev Clin Oncol 2017, 14(12):717-734.

27. Fridman WH, Pages F, Sautes-Fridman C, Galon J: The immune contexture in human tumours: impact on clinical outcome. Nat Rev Cancer 2012, 12(4):298-306.

28. Chen $\mathrm{X}$, Song E: Turning foes to friends: targeting cancer-associated fibroblasts. Nat Rev Drug Discov 2019, 18(2):99-115.

29. Stransky N, Egloff AM, Tward AD, Kostic AD, Cibulskis K, Sivachenko A, Kryukov GV, Lawrence MS, Sougnez $C$, McKenna A et al: The mutational landscape of head and neck squamous cell carcinoma. Science 2011, 333(6046):1157-1160.

30. Zhang C, Chen S, Ma X, Yang Q, Su F, Shu X, Xie W, Feng M, Xiong B: Upregulation of STC2 in colorectal cancer and its clinicopathological significance. Onco Targets Ther 2019, 12:1249-1258.

31. Tamura K, Furihata M, Chung SY, Uemura M, Yoshioka H, liyama T, Ashida S, Nasu Y, Fujioka T, Shuin $\mathrm{T}$ et al: Stanniocalcin 2 overexpression in castration-resistant prostate cancer and aggressive prostate cancer. Cancer Sci 2009, 100(5):914-919.

32. Holloway E: From genotype to phenotype: linking bioinformatics and medical informatics ontologies. Comp Funct Genomics 2002, 3(5):447-450.

33. Finlay CA, Hinds PW, Levine AJ: The p53 proto-oncogene can act as a suppressor of transformation. Cell 1989, 57(7):1083-1093.

34. Kamada R, Toguchi Y, Nomura T, Imagawa T, Sakaguchi K: Tetramer formation of tumor suppressor protein p53: Structure, function, and applications. Biopolymers 2016, 106(4):598-612.

35. Tanaka N, Zhao M, Tang L, Patel AA, Xi Q, Van HT, Takahashi H, Osman AA, Zhang J, Wang J et al: Gain-of-function mutant p53 promotes the oncogenic potential of head and neck squamous cell carcinoma cells by targeting the transcription factors FOXO3a and FOXM1. Oncogene 2018, 37(10):1279-1292.

36. Azulay EE, Cooks T, Elkabets M: Potential oncogenic roles of mutant-p53-derived exosomes in the tumor-host interaction of head and neck cancers. Cancer Immunol Immunother 2020, 69(2):285-292.

37. Peltonen JK, Vahakangas KH, Helppi HM, Bloigu R, Paakko P, Turpeenniemi-Hujanen T: Specific TP53 mutations predict aggressive phenotype in head and neck squamous cell carcinoma: a retrospective archival study. Head Neck Oncol 2011, 3:20.

38. Zhou G, Liu Z, Myers JN: TP53 Mutations in Head and Neck Squamous Cell Carcinoma and Their Impact on Disease Progression and Treatment Response. J Cell Biochem 2016, 117(12):2682-2692.

39. Poeta ML, Manola J, Goldwasser MA, Forastiere A, Benoit N, Califano JA, Ridge JA, Goodwin J, Kenady D, Saunders $\mathrm{J}$ et al: TP53 mutations and survival in squamous-cell carcinoma of the head and neck. N Engl J Med 2007, 357(25):2552-2561. 
40. Jones PA, Baylin SB: The fundamental role of epigenetic events in cancer. Nat Rev Genet 2002, 3(6):415-428.

41. Law AY, Lai KP, Ip CK, Wong AS, Wagner GF, Wong CK: Epigenetic and HIF-1 regulation of stanniocalcin-2 expression in human cancer cells. Exp Cell Res 2008, 314(8):1823-1830.

42. Chu SJ, Zhang J, Zhang R, Lu WW, Zhu JS: Evolution and functions of stanniocalcins in cancer. Int J Immunopathol Pharmacol 2015, 28(1):14-20.

43. Bouras T, Southey MC, Chang AC, Reddel RR, Willhite D, Glynne R, Henderson MA, Armes JE, Venter $D J$ : Stanniocalcin 2 is an estrogen-responsive gene coexpressed with the estrogen receptor in human breast cancer. Cancer Res 2002, 62(5):1289-1295.

44. Esseghir S, Kennedy A, Seedhar P, Nerurkar A, Poulsom R, Reis-Filho JS, Isacke CM: Identification of NTN4, TRA1, and STC2 as prognostic markers in breast cancer in a screen for signal sequence encoding proteins. Clin Cancer Res 2007, 13(11):3164-3173.

45. Buckanovich RJ, Sasaroli D, O'Brien-Jenkins A, Botbyl J, Hammond R, Katsaros D, Sandaltzopoulos R, Liotta LA, Gimotty PA, Coukos G: Tumor vascular proteins as biomarkers in ovarian cancer. J Clin Oncol 2007, 25(7):852-861.

46. Sheikh-Hamad D: Mammalian stanniocalcin-1 activates mitochondrial antioxidant pathways: new paradigms for regulation of macrophages and endothelium. Am J Physiol Renal Physiol 2010, 298(2):F248-254.

47. Huang L, Garcia G, Lou Y, Zhou Q, Truong LD, DiMattia G, Lan XR, Lan HY, Wang Y, Sheikh-Hamad D: Anti-inflammatory and renal protective actions of stanniocalcin-1 in a model of anti-glomerular basement membrane glomerulonephritis. Am J Pathol 2009, 174(4):1368-1378.

48. Kim SJ, Ko JH, Yun JH, Kim JA, Kim TE, Lee HJ, Kim SH, Park KH, Oh JY: Stanniocalcin-1 protects retinal ganglion cells by inhibiting apoptosis and oxidative damage. Plos One 2013, 8(5):e63749.

49. Wang H, Wu K, Sun Y, Li Y, Wu M, Qiao Q, Wei Y, Han ZG, Cai B: STC2 is upregulated in hepatocellular carcinoma and promotes cell proliferation and migration in vitro. BMB Rep 2012, 45(11):629-634.

50. Shi W, Feng L, Dong S, Ning Z, Hua Y, Liu L, Chen Z, Meng Z: Exploration of prognostic index based on immune-related genes in patients with liver hepatocellular carcinoma. Biosci Rep 2020, 40(7).

51. Chen X, Liu Q, Huang W, Cai C, Xia W, Peng Y, Zheng S, Li G, Xu Y, Wang J et al: Stanniocalcin-2 contributes to mesenchymal stromal cells attenuating murine contact hypersensitivity mainly via reducing CD8(+) Tc1 cells. Cell Death Dis 2018, 9(5):548.

\section{Figures}


STC2
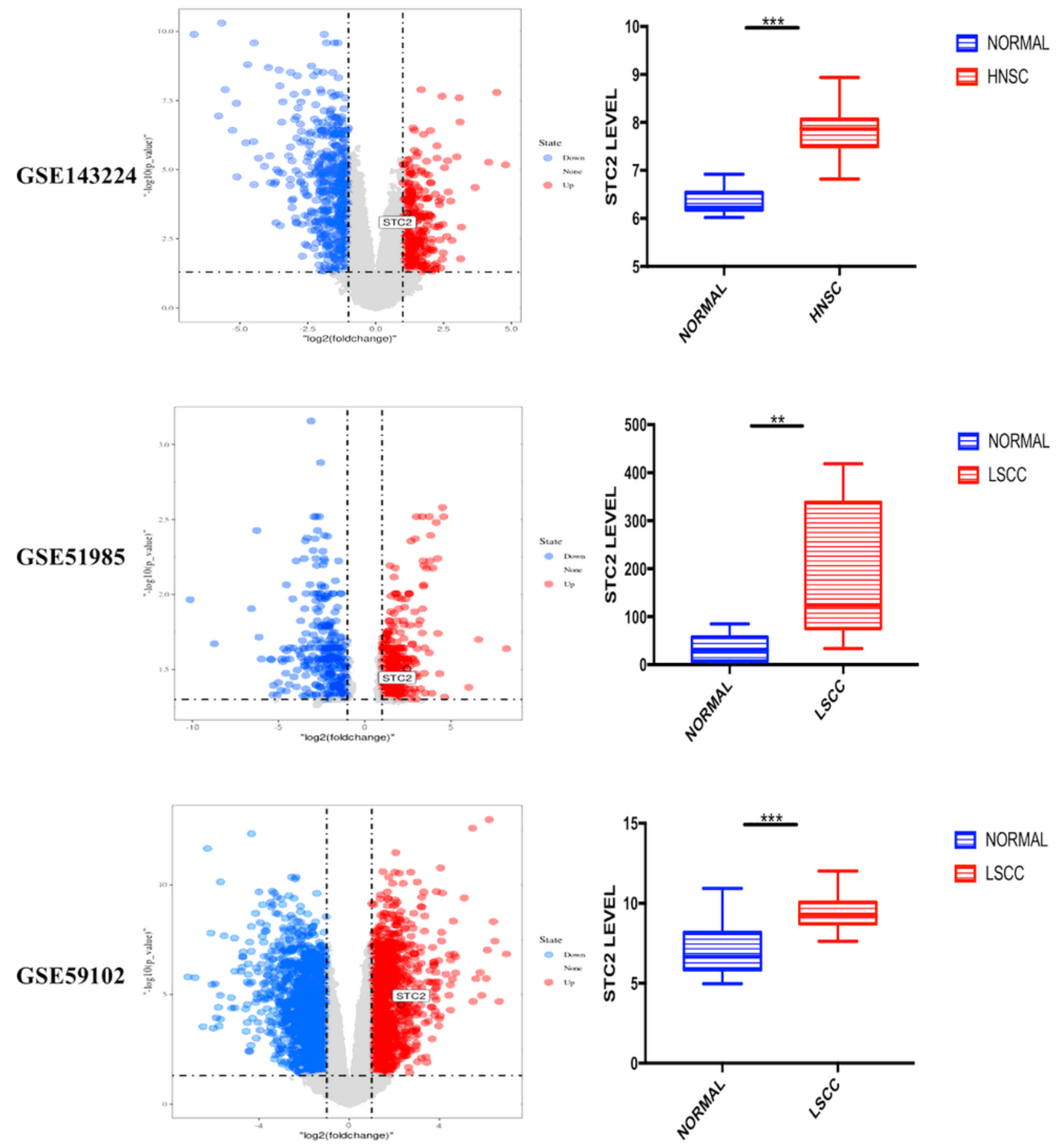

目 LSCC

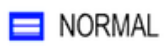

曰

\section{Figure 1}

STC2 gene expression profiles in HNSC (or LSCC) using GSE143112, GSE51985, and GSE59102 datasets. 

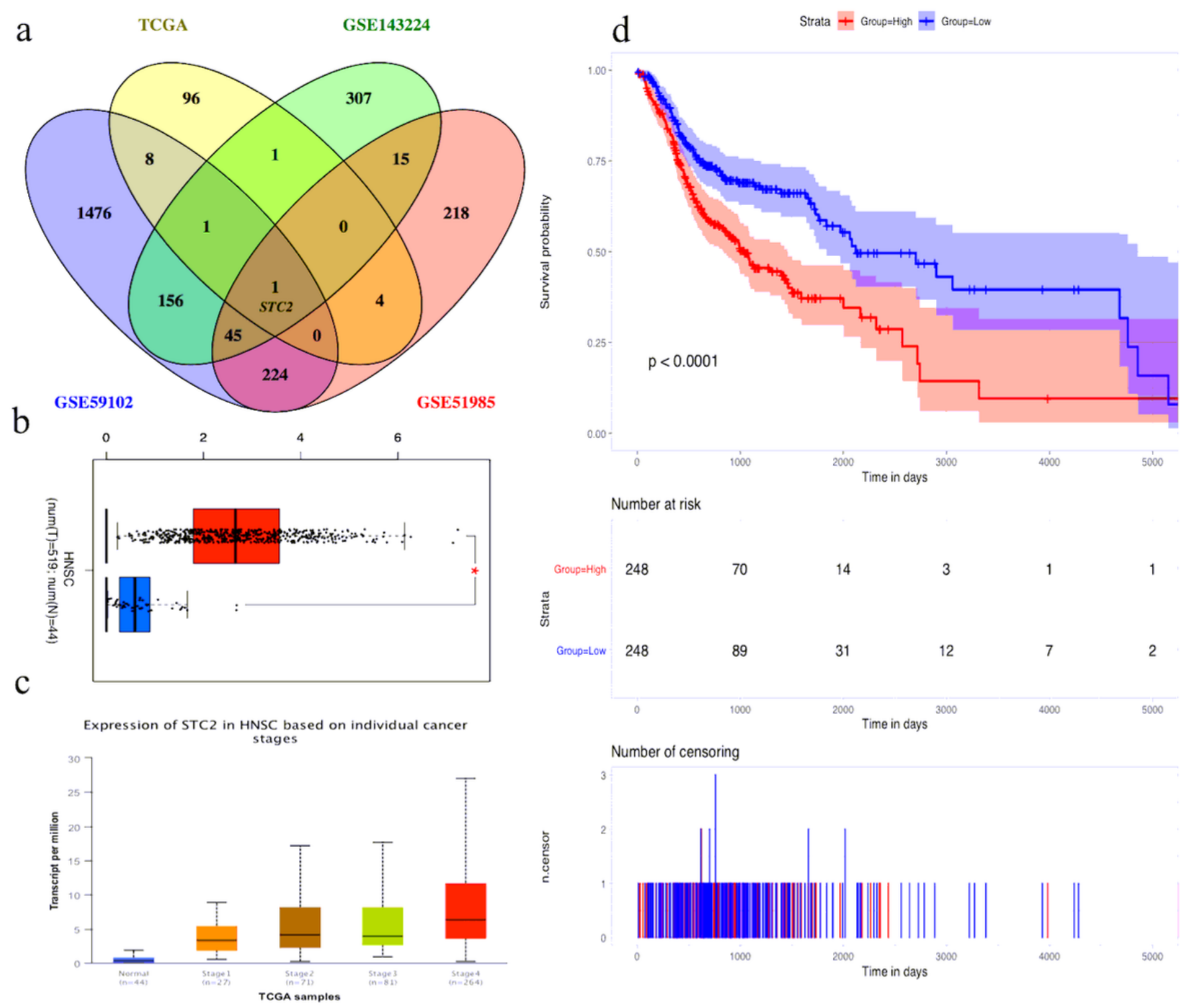

\section{Figure 2}

(a, b, c) STC2 expressions in HNSC with the TCGA cohort using the GEPIA and UALCAN web-tools. (d) The Kaplan-Meier survival curve based on STC2 expression in HNSC. 
a b
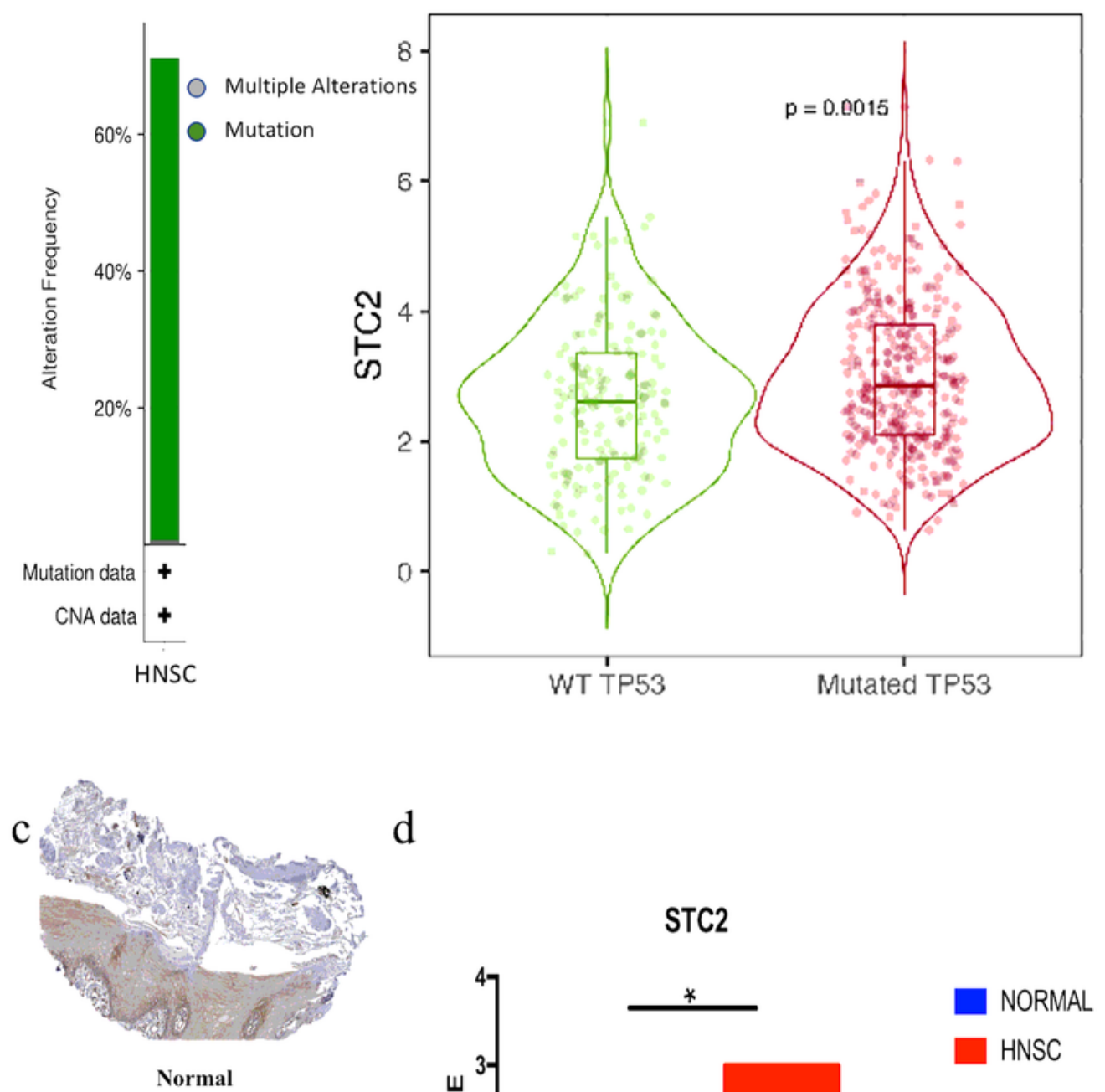

d

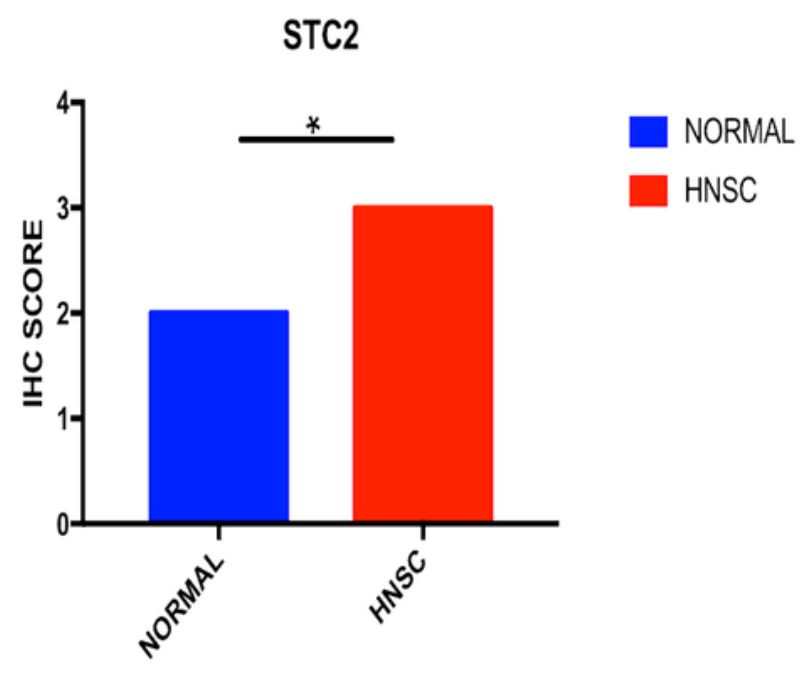

HNSC

\section{Figure 3}

(a) The frequency of TP53 mutation in HNSC. (b) The relationship of STC2 expression with TP53 mutation. (c, d) The intensity of STC2-positive staining in HNSC versus normal tissues. 

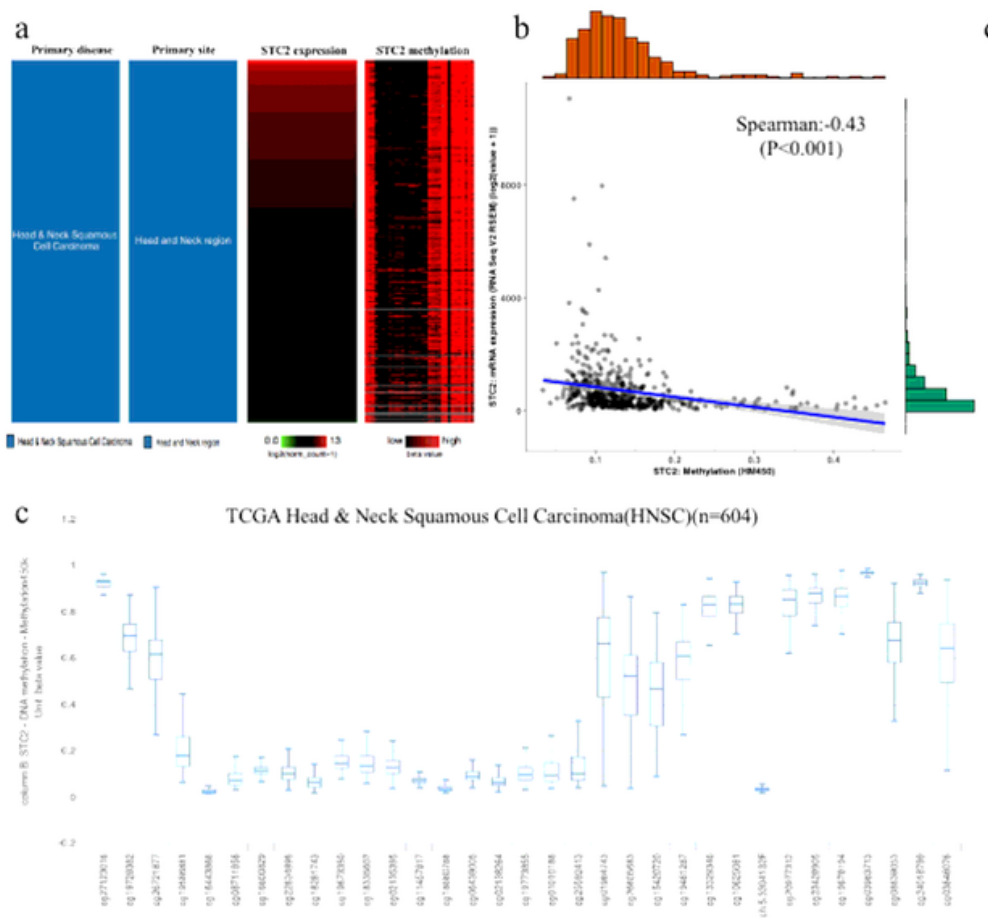

d

\section{Figure 4}

$(a, b)$ The correlation between STC2 expression and STC2 DNA methylation. (c) The methylation levels at 33 CpG sites. (d) Correlation s of individual CpG sites with STC2 expression.

\section{Kaplan Meier DNA methylation}
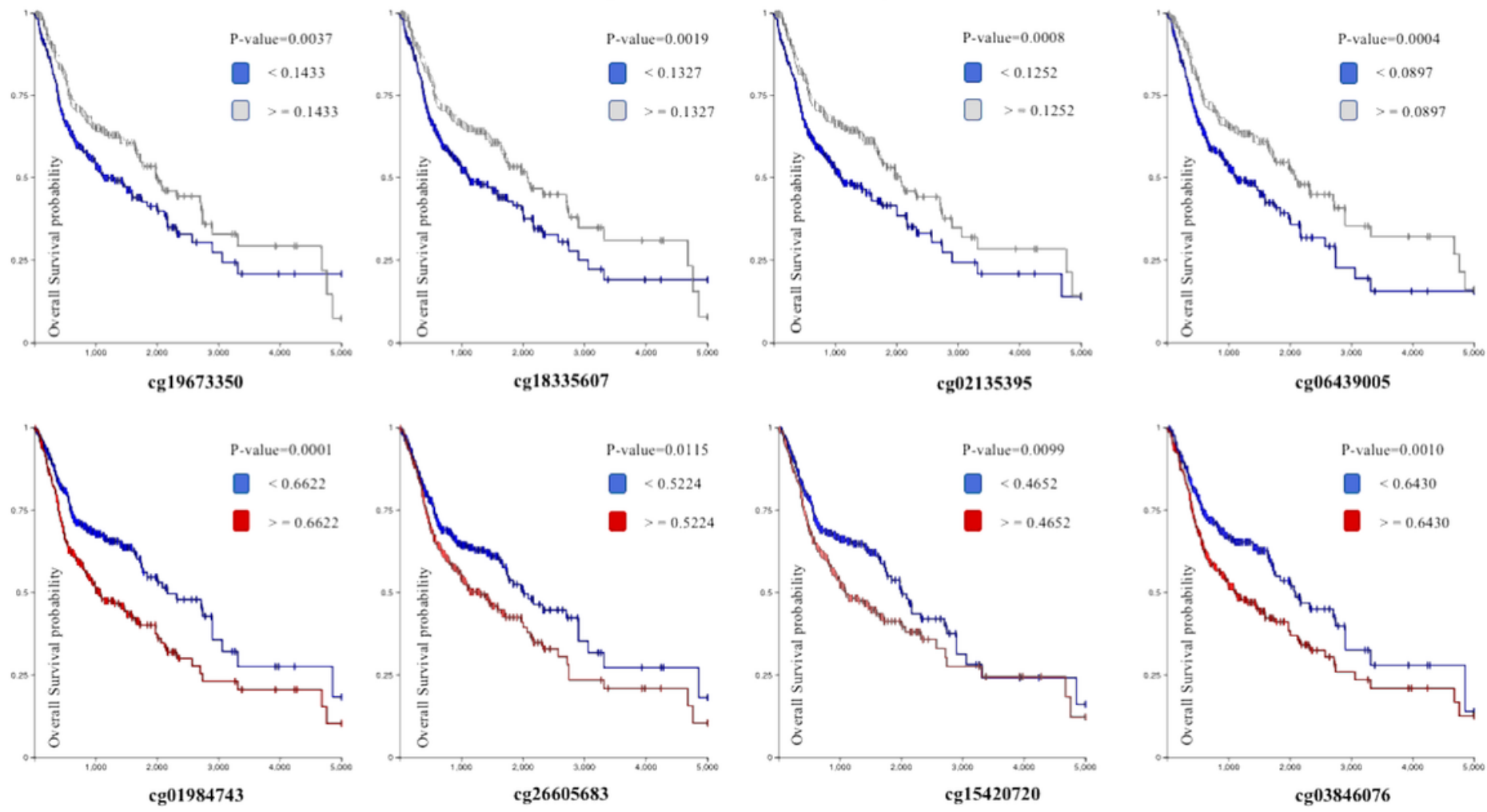

Figure 5 
Kaplan-Meier survival curves based on the methylation levels at eight CpG sites.
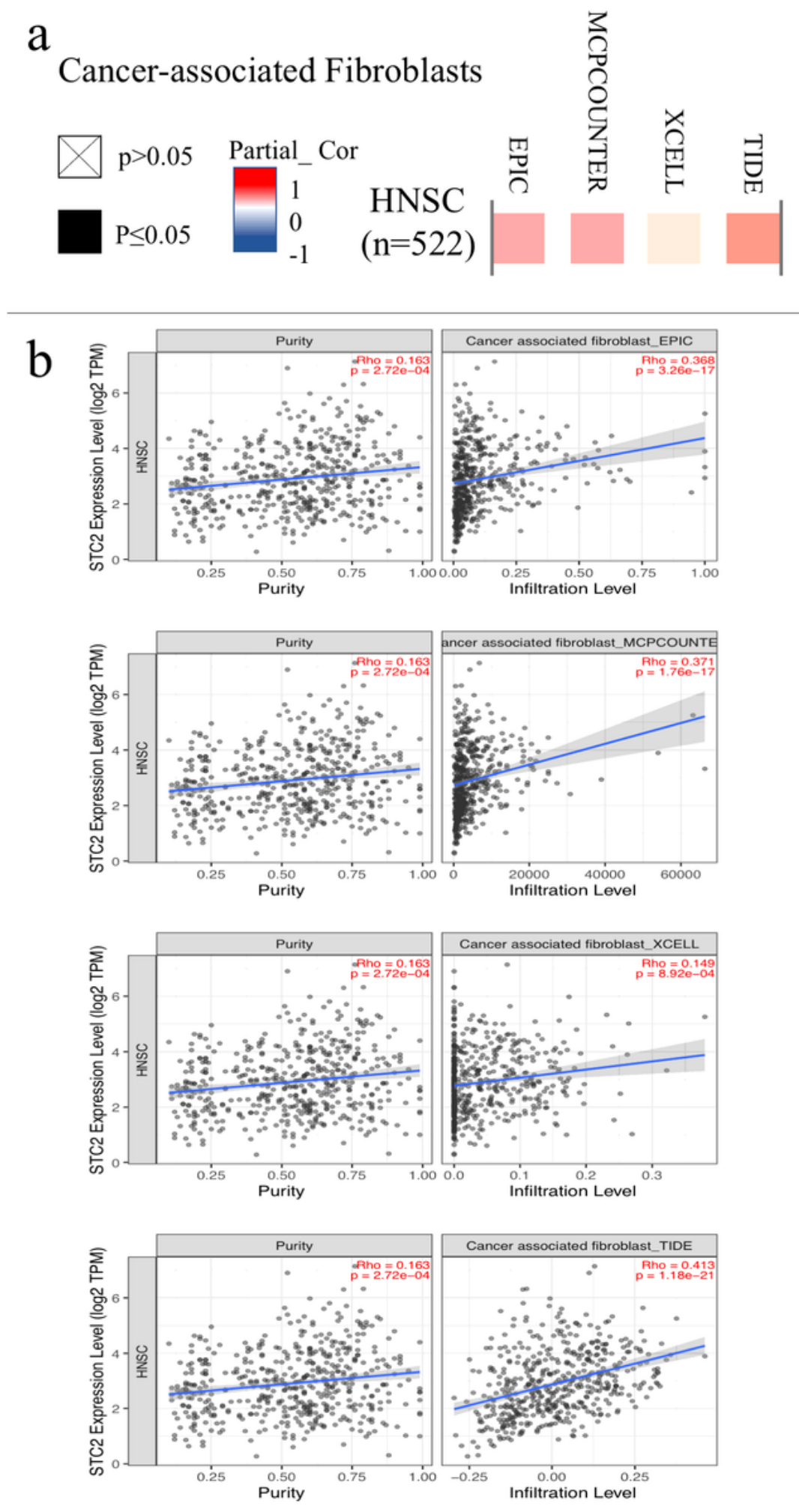

\section{Figure 6}

(a) The correlation between cancer-associated fibroblasts infiltration level with STC2 expression. (b) The EPIC, MCPCOUNTER, XCELL and TIDE algorithms were used to explore the potential relationship between the expression level of the STC2 gene and the infiltration level of cancer-associated fibroblasts. 

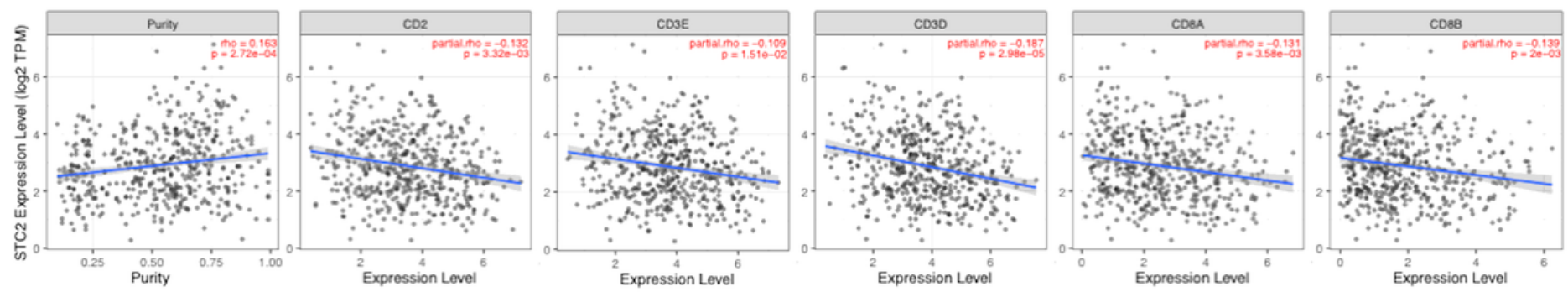

T cell
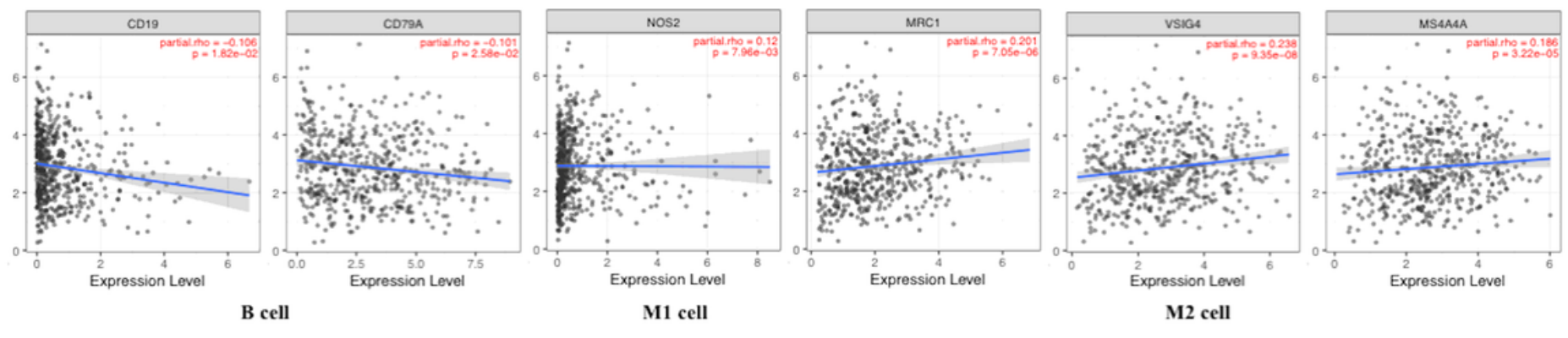

Figure 7

The correlation of STC2 expression with the infiltration levels of tumor-infiltrating immune cells. 

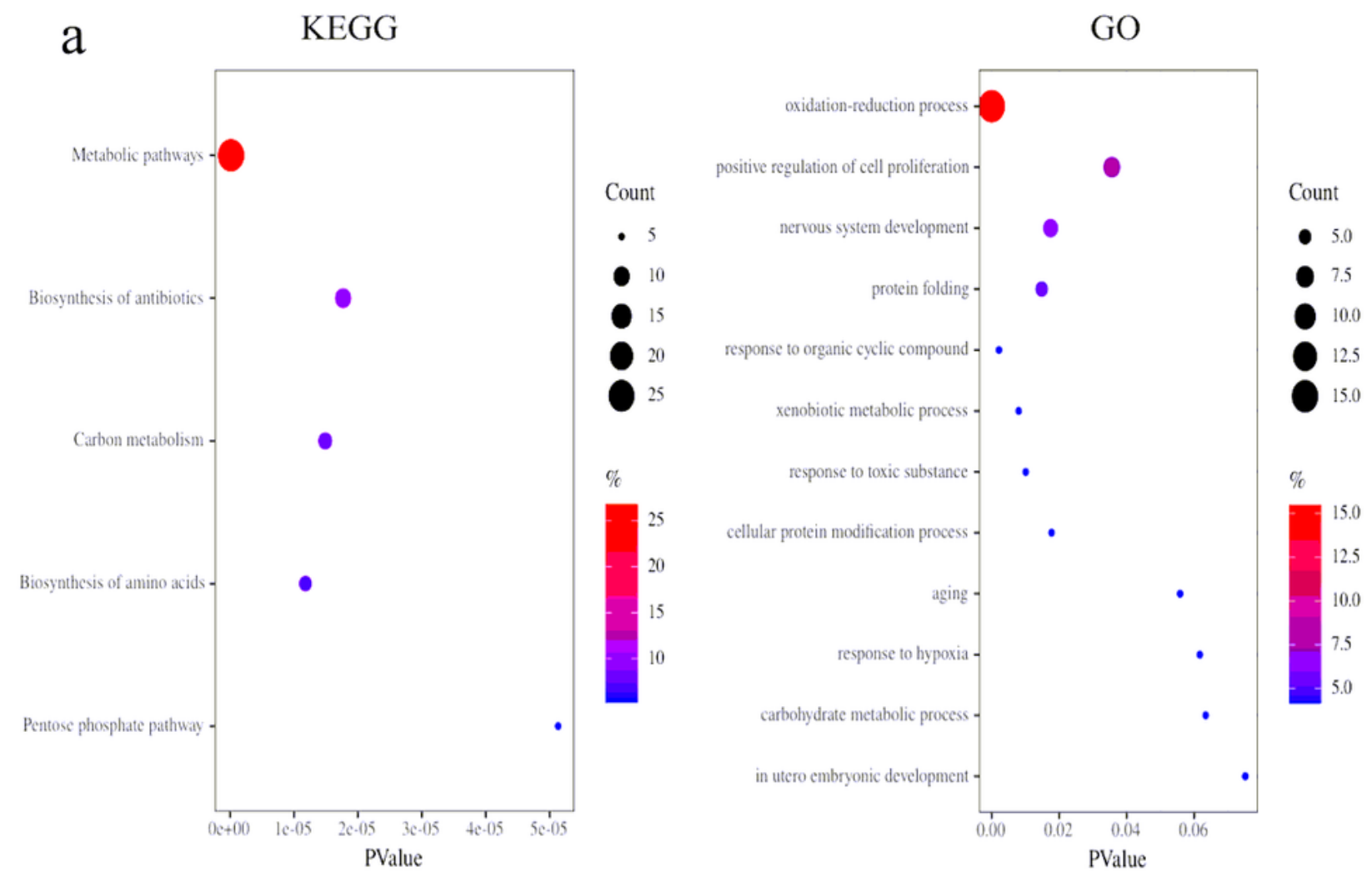

b

UALCAN-Genes positively correlated with STC2 in HNSC

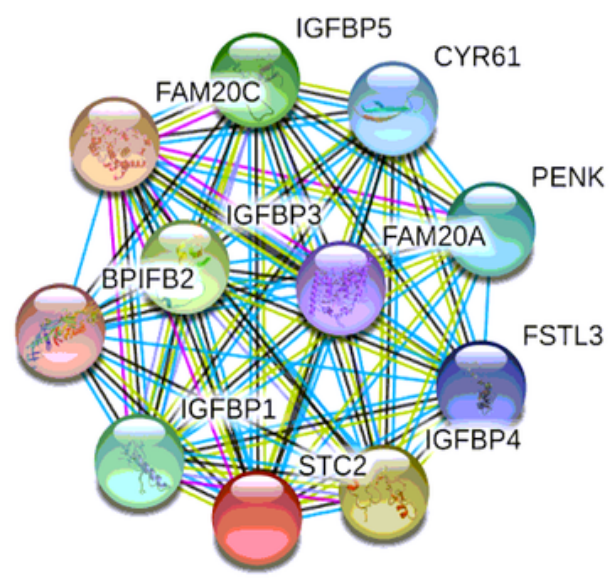

PPI: An interaction score of 0.4 was set as cut-off value. Genes positively correlated with STC2 in HNSC (Genes with extremely low expression (Median TPM $<0.5$ ) are filtered out of the list)

\section{Figure 8}

(a) KEGG and GO enrichment analyses of STC2-related genes. (b) The top 10 proteins significantly interacting with STC2 